JOURNAL OF THE

AMERICAN MATHEMATICAL SOCIETY

Volume 13, Number 3, Pages 639-650

S 0894-0347(00)00336-2

Article electronically published on April 26, 2000

\title{
A NEW PROOF OF THE HOWE CONJECTURE
}

\author{
DAN BARBASCH AND ALLEN MOY
}

\section{INTRODUCTION}

1.1. Let $k$ be a nonarchimedean local field of arbitrary characteristic. Denote by $\mathcal{G}=\mathrm{G}(k)$ the $k$-rational points of a connected reductive algebraic group $\mathrm{G}$ defined over $k$, and let $\mathfrak{g}=\operatorname{Lie}(\mathrm{G})(k)$ denote the $k$-rational points of the Lie algebra. If $\Omega$ is a compact subset of either $\mathcal{G}$ (resp. $\mathfrak{g}$ ), set

$$
{ }^{\mathcal{G}} \Omega:=\{A d(g) w \mid w \in \Omega, g \in \mathcal{G}\} .
$$

Let $J(\Omega)$ denote the space of $\mathcal{G}$-invariant distributions supported on $\operatorname{Ad}(\mathcal{G})(\Omega)$. If $g \in \mathcal{G}$, set $\operatorname{Ad}(g) f: z \mapsto f\left(g^{-1} z g\right)$. If $\phi$ is a locally constant compactly supported function on $\mathcal{G}$ (resp. $\mathfrak{g}$ ), define $\tau_{\Omega}(\phi)$ to be the linear functional on $J(\Omega)$ defined by

$$
\tau_{\Omega}(\phi)(T):=T(\phi) .
$$

The set $\Omega$ will be fixed throughout the paper, so we will abbreviate $\tau_{\Omega}$ to $\tau$. The $\mathcal{G}$-invariance of the distributions in $J(\Omega)$ means $\tau(A d(g) f)=\tau(f)$.

If $L$ is a compact open subgroup of $\mathcal{G}$, denote as $C_{c}(\mathcal{G} / L)$ the space of compactly supported functions on $\mathcal{G}$ which are right $L$-invariant. Similary, if $\mathcal{L}$ is an open compact lattice in $\mathfrak{g}$, let $C_{c}(\mathfrak{g} / \mathcal{L})$ be the space of compactly supported functions on $\mathfrak{g}$ which are $\mathcal{L}$-invariant. In [Ho1], Howe conjectured that if $\Omega$ is a compact subset of $\mathfrak{g}($ resp. $\mathcal{G})$, then the restriction of the distributions in $J(\Omega)$ to $C_{c}(\mathfrak{g} / \mathcal{L})$ (resp. $C_{c}(\mathcal{G} / L)$ ) is finite dimensional.

A more common formulation of the group version of the Howe conjecture replaces the space $C_{c}(\mathcal{G} / L)$ with the Hecke algebra $\mathcal{H}(\mathcal{G} / / L)$ of compactly supported $L$-biinvariant functions on $\mathcal{G}$. Since $\mathcal{H}(\mathcal{G} / / L) \subset C_{c}(\mathcal{G} / L)$, apriori, our formulation with $C_{c}(\mathcal{G} / L)$ implies the Hecke algebra formulation. It is a simple matter to show that in fact the two formulations are equivalent. If $f \in C_{c}(\mathcal{G} / L)$, then the function $F(g):=\int_{L} f\left(k g k^{-1}\right) d k$ belongs to the Hecke algebra, and the map $f \mapsto F$ is a surjection. The linear functional on $J(\Omega)$ induced by $f$ is a positive multiple of the linear functional induced by $F$. Thus, the two spaces of linear functionals are equal and so the two formulations are equivalent.

1.2. In Ho2, Howe proved (for arbitrary characteristic) the algebra version of the conjecture for $G L(n)$. For purposes of comparison, we summarize Howe's argument. Let $\mathcal{R}$ denote the ring of integers of $k$, and let $\varpi$ denote a generator of the prime ideal. By a homogeneity property, we can take $\mathcal{L}=\mathfrak{g l}(n, \mathcal{R})$. A basis for $C_{c}(\mathfrak{g l}(n) / \mathcal{L})$ is given by the characteristic functions $1_{X+\mathcal{L}}$ of the cosets of

Received by the editors April 20, 1999 and, in revised form, March 7, 2000.

2000 Mathematics Subject Classification. Primary 22E35.

The authors were supported in part by the National Science Foundation grants DMS-9706758 and DMS-9801264. 
$\mathcal{L}$ in $\mathfrak{g l}(n)$. As we have already indicated, the Howe conjecture is equivalent to the statement that the linear functionals of $J(\Omega)$ induced by the functions $1_{X+\mathcal{L}}$, i.e. $\tau\left(1_{X+\mathcal{L}}\right)(T):=T\left(1_{X+\mathcal{L}}\right)$, is a finite-dimensional space. The lattices $\varpi^{-i} \mathcal{L}$, $i \in \mathbb{Z}$, filter $\mathfrak{g l}(n)$. Howe shows there is a positive $N=N(\Omega)$ so that if $i>N$ and $X \in \varpi^{-i} \mathcal{L}-\varpi^{-i+1} \mathcal{L}$, then $\tau\left(1_{X+\mathcal{L}}\right)$ is a linear combination of the linear functionals $\tau\left(1_{Z+\mathcal{L}}\right)$ with $Z \in \varpi^{-i+1} \mathcal{L}$. So, by induction $\tau\left(1_{X+\mathcal{L}}\right)$ lies in the finite-dimensional span of the functionals $\tau\left(1_{Z+\mathcal{L}}\right)$ with $Z \in \varpi^{-N} \mathcal{L}$. To accomplish this, Howe makes a reduction to the situation when the coset $X+\mathcal{L}$ contains a nilpotent element. In this situation, Howe then uses two steps. A first step, which we refer to as the descent step, involves conjugation of the coset $X+\mathcal{L}$ by a diagonal element $d$ so that $A d(d)(X+\mathcal{L}) \subset \varpi^{-i+1} \mathcal{L}$; in particular, the descent step lowers the 'level' of the coset. However this step distorts the lattice $\mathcal{L}$ to $\mathcal{L}_{d}:=\operatorname{Ad}(d)(\mathcal{L})$. In other words, the result is not an $\mathcal{L}$-invariant set. To correct this, Howe uses a rather involved argument which we call the regeneration step. More precisely, he shows that if $Y \in \varpi^{-i+1} \mathcal{L}$, then there are $X_{j}+\mathcal{L} \subset \varpi^{-i+1} \mathcal{L}$ and $g_{k}, h_{j} \in G$ such that

$$
\sum_{k} A d\left(g_{k}\right) 1_{Y+\mathcal{L}_{d}}=\sum_{j} A d\left(h_{j}\right) 1_{X_{j}+\mathcal{L}} .
$$

In [HC], Harish-Chandra extended Howe's method, when the characteristic is zero, to arbitrary reductive Lie algebras. Harish-Chandra then used the conjecture, in both its Lie algebra and group formulations, as a fundamental underpinning of his approach to harmonic analysis on the group and Lie algebra. Many properties of $\mathcal{G}$ invariant distributions, which for real Lie groups follow from differential equations, in the $p$-adic case are consequences of the Howe conjecture and other facts, e.g. properties of orbital integrals. Clozel proved the group Howe conjecture in [C] for characteristic zero via a method very different from Howe's and Harish-Chandra's descent method. Our goal here is to give a new proof of the group Howe conjecture via the Bruhat-Tits building. A key tool in our proof is the geodesic convexity of the displacement function. Highlights of the proof are that it is valid in all characteristics, and it has similarities to Howe's and Harish-Chandra's method as well as to the existence proof of an unrefined minimal K-type.

1.3. We formulate and sketch our proof of the group Howe conjecture. The group $\mathcal{G}$ acts polysimplicially isometrically on the Bruhat-Tits building $\mathcal{B}$. Suppose $x \in \mathcal{B}$. For $r \in \mathbb{N}=\{1,2, \ldots\}$, let $\mathcal{G}_{x, r}$ denote the subgroup of $\mathcal{G}$ defined in [MP]. As $r$ varies over $\mathbb{N}$, the groups $\mathcal{G}_{x, r}$ form a fundamental system of neighborhoods of the identity.

1.4. Theorem (Howe Conjecture). Suppose $\Omega$ is a compact subset of $\mathcal{G}$. The space of linear functionals on $J(\Omega)$ induced by the elements of $C_{c}\left(\mathcal{G} / \mathcal{G}_{x, r}\right)$ is finite dimensional.

By a simple property of the groups $\mathcal{G}_{x, r}$, we can assume $x$ is an interior point of some closed chamber $C$. If $g \in \mathcal{G}$, let $d_{g}$ denote the displacement function of $g$. Displacement functions allow us to filter $C_{c}\left(\mathcal{G} / \mathcal{G}_{x, r}\right)$ by finite-dimensional subspaces $C_{c}\left(\mathcal{G} / \mathcal{G}_{x, r}\right)_{N}, N \geq 0$, which exhaust $C_{c}\left(\mathcal{G} / \mathcal{G}_{x, r}\right)$. We then show, using convexity properties of displacement functions (notably 2.2 and 2.4), that for sufficiently large $N$, the space of linear functionals on $J(\Omega)$ induced by $C_{c}\left(\mathcal{G} / \mathcal{G}_{x, r}\right)_{N}$ stabilizes. The descent step in our proof occurs precisely when a set displacement function $d_{g \mathcal{G}_{x, r}}$ has minimal value $d_{C}(g)$ on $C$ which is strictly greater than its global minimum. 
In this situation, we know apriori that there is a chamber $D$ meeting $C$ and a point $y \in D^{o}$, so that $d_{g \mathcal{G}_{x, r}}(y)<d_{C}(g)$. Then, under certain conditions, which we can assure are satisfied, the regeneration step is statement (3.5.3) that the union of a suitable collection of conjugates of $g \mathcal{G}_{x, r}$ is a union of $\mathcal{G}_{y, r}$ cosets. An essential difference (besides our use of the Bruhat-Tits building) between our approach and that of Howe and Harish-Chandra is the regeneration step. Howe and HarishChandra use properties of nilpotent elements to accomplish the regeneration step while our proof, in particular 3.5.1, makes use of the Bruhat decomposition and is considerably simpler.

After completing a preliminary version of this manuscript, we became aware of a generalization of Howe's conjecture for weighted orbital integrals made and proved by Arthur in A1, A2. Our main result applies to this situation as well. This is the subject of the final section.

\section{Preliminaries on Displacement functions}

2.1. We recall some notation and facts from [MP], $[\mathrm{BT}]$ and [M]. Let $k$ denote a nonarchimedean local field of arbitrary characteristic, and let $\mathrm{G}$ be a connected reductive algebraic group defined over $k$. Let $\mathcal{D} G$ be the derived group of $\mathrm{G}$, and let $\mathcal{B}=\mathcal{B}(\mathcal{D} G, k)$ be the Bruhat-Tits buildings of $\mathcal{D} G / k$. If the root system of $\mathcal{D} G$ is irreducible, the building is a simplicial complex. More generally, the building is a polysimplicial complex in which a polysimplex is a product of simplicies in the buildings of the irreducible components of the root system. The building is also a metric space. The group $\mathcal{G}=\mathrm{G}(k)$ acts both polysimplicially and isometrically on the building. Denote as $\operatorname{Aut}(\mathcal{B})$ the group of polysimplicial isometries of $\mathcal{B}$.

Suppose $\mathrm{S}$ is a maximal $k$-split torus of $\mathrm{G}$. Let $A=A(S)$ denote the apartment in $\mathcal{B}$ associated to the torus $S$. Let $Z$ denote the centralizer of $S$ in $G$, and let $N$ denote the normalizer of $\mathbf{Z}$. Both $\mathbf{Z}$ and $\mathbf{N}$ are defined over $k$. The group $\mathcal{N}=\mathbf{N}(k)$ acts on the apartment $A$.

Let $\Psi=\{\psi\}$ be the affine root system on $A$. We choose a normalization of the valuation on $k$, hence a distance on $\mathcal{B}$, so that if $\psi$ is a (nonconstant) affine root, then so is $\psi \pm 1$ (see [BT, $\S 6.2 .23]$ ). For $\psi \in \Psi$, denote by $H_{\psi}$ the vanishing hyperplane $H_{\psi}:=\{v \in A \mid \psi(v)=0\}$. Two points $x, y \in A$ belong to the same facet in $A$ and hence $\mathcal{B}$ precisely when $x$ and $y$ never belong to opposite sides of an affine hyperplane $H_{\psi}$. The closure of a facet is a polysimplex. A chamber $C$ is a polysimplex of maximal dimension. If $F$ is a facet, let $\operatorname{Stab}(F)$ denote the subgroup of $\mathcal{G}$ which fixes the points of $F$ pointwise, and denote as $\mathcal{G}_{F}$ the maximal compact subgroup of $\operatorname{Stab}(F)$. Recall:

i) The group $\mathcal{N}$ acts as a group of polysimplicial isometries of the Euclidean space $A$, and the action is transitive on chambers.

ii) The group $\mathcal{G}$ has the Bruhat decomposition $\mathcal{G}=\mathcal{G}_{C} \mathcal{N} \mathcal{G}_{C}$.

If $x$ and $y$ are two points of the building, denote as dist $(x, y)$ the distance between $x$ and $y$. The displacement function $d_{g}$ of an element $g \in \operatorname{Aut}(\mathcal{B})$ is the function $d_{g}: \mathcal{B} \rightarrow \mathbb{R}$ defined as

$$
d_{g}(x):=\operatorname{dist}(x, g x) .
$$

If $\Xi$ is a bounded nonempty subset of $\mathcal{G}$, let

$$
d_{\Xi}(y):=\sup \left\{d_{g}(y) \mid g \in \Xi\right\} .
$$

We call $d_{\Xi}$ the set displacement function of the set $\Xi$. 
Finally, let

$$
d(g):=\min \left\{d_{g} \mid g \in \mathcal{G}\right\} .
$$

The next theorem summarizes results from [M]. Recall $\mathcal{B}=\mathcal{B}(\mathcal{D} G, k)$.

2.2. Theorem (Convexity of the displacement function).

i) The displacement function $d_{g}$ is geodesically convex for any $g \in \operatorname{Aut}(\mathcal{B})$.

ii) Suppose $\Xi$ is a bounded set in $\mathcal{G}$. Then $d_{\Xi}$ is a convex function and it achieves a minimum on $\mathcal{B}$.

iii) The function $g \mapsto d(g)$ is a continuous class function on $\mathcal{G}$.

Suppose $C$ is a chamber contained in the apartment $A=A(S)$. If $g \in \mathcal{G}$, let $g=i_{1} n i_{2}, i_{1}, i_{2} \in \mathcal{G}_{C}$ and $n \in \mathcal{N}$, be a Bruhat decomposition of the element $g$. The elements $i_{1}$ and $i_{2}$ fix every point $x \in C$; therefore

$$
\begin{aligned}
d_{g}(x) & =\operatorname{dist}(x, g x)=\operatorname{dist}\left(x, i_{1} n i_{2} x\right)=\operatorname{dist}\left(x, i_{1} n x\right) \\
& =\operatorname{dist}\left(i_{1}^{-1} x, n x\right)=\operatorname{dist}(x, n x)=d_{n}(x) .
\end{aligned}
$$

Thus, the displacement functions $d_{g}$ and $d_{n}$ have the same restriction on the chamber $C$. If $\bar{n} \in \mathcal{N} /\left(\mathcal{N} \cap \mathcal{G}_{C}\right)$, take $n \in \mathcal{N}$ to be a representative of $\bar{n}$. If $n_{1}$ and $n_{2}$ are representatives of $\bar{n}$, then on $A, d_{n_{1}}=d_{n_{2}}$. Denote as

$$
d_{C}(g):=\text { the minimal value of } d_{g} \text { on } C \text {. }
$$

2.3. Lemma. i) Along a geodesic path in $\mathcal{B}$, the displacement function $d_{g}$ is piecewise differentiable.

ii) Let $C$ be a chamber in the apartment $A$. The set of values $d_{C}(n), n \in \mathcal{N}$, hence the set of values $d_{C}(g), g \in \mathcal{G}$, below a given value $R$ is finite.

2.4. Proposition. Suppose $g \in \mathcal{G}$ and $x \in \mathcal{B}$. Set $\ell=d_{g}(x)$. If $\ell>d(g)$, then $d_{g}<\ell$ on the open interval $(x, g x)$.

Proof. We observe that $d_{g}(g x)=\operatorname{dist}\left(g x, g^{2} x\right)=\operatorname{dist}(x, g x)=d_{g}(x)=\ell$. We make two assertions.

i) $d_{g} \leq \ell$ along the geodesic segment $[x, g x]$.

ii) $d_{g}$ is not constant along $[x, g x]$.

The first assertion is trivially a consequence of our observation that $d_{g}(g x)=$ $d_{g}(x)=\ell$ and the convexity of $d_{g}$ on $[x, g x]$. To prove assertion ii), suppose by way of contradiction that $d_{g}$ is constant along $[x, g x]$. Then clearly $d_{g}=\ell$ on $[x, g x]$. Let $y$ be the midpoint of $[x, g x]$. Then, $g y$ is the midpoint of the geodesic $\left[g x, g^{2} x\right]$. If $d_{g}$ is constant along $[x, g x]$, then $d_{g}(y)=\ell$, i.e. the geodesic segment $[y, g y]$ has length $\ell$, and so both $\operatorname{dist}(y, g x)=\frac{\ell}{2}$ and $\operatorname{dist}(g x, g y)=\operatorname{dist}(x, y)=\frac{\ell}{2}$. We conclude that the point $g x$ must be the midpoint of the geodesic segment $[y, g y]$. We then deduce that the curve consisting of $[x, g x]$ joined to $\left[g x, g^{2} x\right]$ is a geodesic curve in a neighborhood of the point $g x$ and hence must be a geodesic curve. An obvious induction allows us to conclude that for all $i \in \mathbb{Z}$, the geodesic segments $\left[g^{i} x, g^{i+1} x\right]$ piece together to form a geodesic. Choose $z$ so that $d_{g}(z)<\ell$. Then, for $j$ a positive integer, $\operatorname{dist}\left(z, g^{j} z\right) \leq j \operatorname{dist}(z, g z)$ and so

$j \ell=\operatorname{dist}\left(x, g^{j} x\right) \leq \operatorname{dist}\left(z, g^{j} z\right)+\operatorname{dist}(x, z)+\operatorname{dist}\left(g^{j} z, g^{j} x\right) \leq j d_{g}(z)+2 \operatorname{dist}(x, z)$.

These inequalities lead to a contradiction to $d_{g}(z)<\ell$ provided $j$ is sufficiently large. We therefore conclude that the displacement function is not constant along the geodesic $[x, g x]$. The assertion $d_{g}<\ell$ on the open interval $(x, g x)$ is now a simple consequence of assertions i), ii), Lemma $2.3 \mathrm{i}$ ) and the convexity of $d_{g}$. 
2.5. Corollary. Suppose $g \in G$ and $C$ is a chamber. Let $z$ be a point in $C$ with $d_{g}(z)=d_{C}(g)$. If $d_{C}(g)>d(g)$, then $[z, g z] \cap C=\{z\}$.

\section{Proof of the Howe Conjecture}

3.1. Recall that we have normalized the valuation so that if $\psi$ is a (nonconstant) affine root, then so is $\psi \pm 1$. For $x \in \mathcal{B}$ and $r$ a positive integer, define the open compact subgroup $\mathcal{G}_{x, r}$ as in [MP]. Since $r>0$, the group $\mathcal{G}_{x, r}$ fixes a neighborhood $U$ of $x$ and therefore $d_{g \mathcal{G}_{x, r}}$ equals $d_{g}$ on $U$. The convex function $d_{g \mathcal{G}_{x, r}}$ has a local, hence global, minimum at the point $x$ if and only if the same holds for $d_{g}$.

3.2. Proposition. i) Suppose $r \in \mathbb{N}$ and $F$ is a facet. If $\psi$ is an affine root, then on $F$, either $\psi>r$, or $\psi<r$, or $\psi$ is identically $r$. In particular, $\mathcal{G}_{x, r}=\mathcal{G}_{y, r}$ for all $x, y \in F$.

ii) Suppose $F$ and $E$ are two facets in $\mathcal{B}, E$ is contained in the closure of $F$ and $y \in E$ and $x \in F$. Let $A=A(S)$ be an apartment containing $F$. Then

(1) $\mathcal{G}_{y, r} \supset \mathcal{G}_{x, r}$

(2) $\mathcal{G}_{y, r}=\mathcal{G}_{x, r} \Pi^{\prime} U_{\psi}$, where the product $\Pi^{\prime}$ is over those affine roots $\psi$ of $A$ which are less than $r$ on $F$ and equal to $r$ on $E$.

Proof. To prove assertion i), we argue by contradiction. Suppose there is an affine root $\psi$ which is nonconstant on $F$ and takes values both greater and less than $r$. Then, the vanishing wall of the affine root $\psi-r$ properly divides the facet $F$, a contradiction. To prove ii), we argue as follows. If $E$ is a facet contained in the closure of the facet $F$, then clearly if the values of an affine root on the facet $F$ are $\geq r$, then its values on the facet $E$ are also $\geq r$. Assertions ii.1) and ii.2) follow immediately from this observation.

3.3. Preliminary remarks on the proof of the Howe Conjecture. Recall our notation: The set $\Omega$ is a compact subset of $\mathcal{G}$, and $J(\mathcal{G})$ is the space of $\mathcal{G}$-invariant distributions supported on $\Omega$. If $f \in C_{c}(\mathcal{G})$, then $\tau(f)$ denotes the linear functional on $J(\Omega)$ defined as $\tau(f)(T):=T(f)$. Also, $x \in \mathcal{B}$ and $r \in \mathbb{N}$.

We make two elementary remarks. The first is that, as an immediate consequence of Proposition 3.2, we have

i) $C_{c}\left(\mathcal{G} / \mathcal{G}_{x, r}\right)=C_{c}\left(\mathcal{G} / \mathcal{G}_{y, r}\right)$ when $x$ and $y$ belong to the same facet,

ii) $C_{c}\left(\mathcal{G} / \mathcal{G}_{y, r}\right) \subset C_{c}\left(\mathcal{G} / \mathcal{G}_{x, r}\right)$ if $y$ lies in the closure of the facet containing $x$.

Therefore it suffices to prove the Howe Conjecture when $x$ belongs to the interior of a chamber $C$. We assume this. To state the second remark, if $X$ is a subset of the closure of some facet, let $\operatorname{Star}(X)$ denote the union of all chambers containing $X$. It is elementary that there exists a constant $R>0$, independent of $x$, so that for any $r \geq R$, the group $\mathcal{G}_{x, r}$ fixes all chambers meeting $\operatorname{Star}(x)$. Our second elementary remark is that it sufficies to prove the Howe Conjecture when $r \geq R$. So, we assume this.

The space $C_{c}\left(\mathcal{G} / \mathcal{G}_{x, r}\right)$ is spanned by the functions

$$
1_{g, x}:=\text { the characteristic function of the coset } g \mathcal{G}_{x, r}
$$

as $g$ varies over $\mathcal{G}$. For $N \geq 0$, set

$$
C_{c}\left(\mathcal{G} / \mathcal{G}_{x, r}\right)_{N}:=\text { the subspace spanned by the elements } 1_{g, x} \text { with } d_{C}(g) \leq N \text {. }
$$

Trivially, if $N_{1} \leq N_{2}$, then $C_{c}\left(\mathcal{G} / \mathcal{G}_{x, r}\right)_{N_{1}} \subset C_{c}\left(\mathcal{G} / \mathcal{G}_{x, r}\right)_{N_{2}}$. 
3.3.2. Proposition. Suppose $r \in \mathbb{N}$ and $\Omega$ is a nonempty compact subset of $\mathcal{G}$. For any $N>0$, there are only finitely many cosets $g \mathcal{G}_{x, r}$ such that $d_{C}(g) \leq N$ and $g \mathcal{G}_{x, r} \cap{ }^{\mathcal{G}} \Omega \neq \varnothing$. In particular, the linear space $\tau\left(C_{c}\left(\mathcal{G} / \mathcal{G}_{x, r}\right)_{N}\right)$ is finite dimensional.

Proof. The kernel of the action of $\mathcal{G}$ on $\mathcal{B}$ is the center $\mathcal{Z}$ of $\mathcal{G}$. When $\mathrm{G}$ is semisimple, $\mathcal{Z}$ is compact and there are only finitely many cosets $g \mathcal{G}_{x, r}$ satisfying $d_{C}(g) \leq N$. Thus, $\tau\left(C_{c}\left(\mathcal{G} / \mathcal{G}_{x, r}\right)_{N}\right)$ is finite dimensional. More generally, let $X(\mathcal{G})$ denote the free abelian group, of rank the difference of the reductive and semisimple $k$-ranks of $\mathrm{G}$, whose elements are the compositions of the $k$-rational characters of $\mathrm{G}$ with the valuation map $k^{\times} \rightarrow \mathbb{Z}$. Define $\beta: \mathcal{G} \rightarrow \operatorname{Hom}(X(\mathcal{G}), \mathbb{Z})$ to be the homomorphism $\beta(g)(\chi):=\chi(g)$. It is clear that $\beta$ is a class function and that $\mathcal{G}^{1}:=\operatorname{ker}(\beta)$ is an open subgroup containing all compact subgroups of $\mathcal{G}$. Because $\Omega$ is compact, $\beta\left({ }^{\mathcal{G}} \Omega\right)$ is a finite set. The condition $g \mathcal{G}_{x, r} \cap{ }^{\mathcal{G}} \Omega \neq \varnothing$ becomes $\beta\left(g \mathcal{G}_{x, r}\right) \in \beta\left({ }^{\mathcal{G}} \Omega\right)$. Combined with the condition $d_{C}(g) \leq N$, these facts imply the first claim of the Proposition. The finite dimensionality follows from the observation that if $\tau\left(1_{g, x}\right)$ is nonzero, then necessarily $g \mathcal{G}_{x, r} \cap \mathcal{G}_{\Omega} \neq \varnothing$.

It is useful for us to observe that Lemma 2.3 ii) implies that the set of values $\left\{d_{C}(g)\right\}$ (which is independent of the choice of $C$ ) is enumerable according to size. Let $0=s_{0}<s_{1}<s_{2}<\cdots<s_{m}<\cdots$ denote the elements of this set. In particular, if $s_{m}<N<s_{m+1}$, then $\tau\left(C_{c}\left(\mathcal{G} / \mathcal{G}_{x, r}\right)_{s_{m}}\right)=\tau\left(C_{c}\left(\mathcal{G} / \mathcal{G}_{x, r}\right)_{N}\right)$. To complete our proof of the Howe Conjecture it is enough to show that $\tau\left(C_{c}\left(\mathcal{G} / \mathcal{G}_{x, r}\right)_{s_{m}}\right)=$ $\tau\left(C_{c}\left(\mathcal{G} / \mathcal{G}_{x, r}\right)_{s_{m+1}}\right)$ when $s_{m}$ is sufficiently large. To do this, we treat the functionals $\tau\left(1_{g, x}\right)$ according to two cases:

i) $d_{C}(g)=d(g)$,

ii) $d_{C}(g)>d(g)$.

Recall that we assume $x \in C^{o}$ (the interior of $C$ ) and $r \geq R$.

3.4. Case $d_{C}(g)=d(g)$. Recall that Theorem 2.2 iii) asserts that the function $d: \mathcal{G} \rightarrow \mathbb{R}$ defined in (2.1.3) is a continuous class function. Denote by $M(\Omega)$ the maximal value achieved by $d$ on $\Omega$. A necessary condition for $\tau\left(1_{g, x}\right) \neq 0$ is that $g \mathcal{G}_{x, r} \cap{ }^{\mathcal{G}} \Omega \neq \varnothing$. Choose $h \in g \mathcal{G}_{x, r} \cap{ }^{\mathcal{G}} \Omega$. Since $d_{h}=d_{g}$ on $C$, we get

$$
d_{C}(h)=d_{C}(g)=d(g) \leq M(\Omega) .
$$

By Proposition 3.3.2 there are only finitely many cosets $g \mathcal{G}_{x, r}$ satisfying $d_{C}(g) \leq$ $M(\Omega)$ and $g \mathcal{G}_{x, r} \cap{ }^{\mathcal{G}} \Omega \neq \varnothing$.

3.5. Case $d_{C}(g)>d(g)$. If $B$ and $C$ are two chambers in the building, denote by $\mathcal{C}(B, C)$ the smallest convex union of chambers which contains both chambers. Equivalently, $\mathcal{C}(B, C)$ is the intersection of all the apartments containing $B$ and $C$.

Choose an apartment $A=A(S)$ containing $C$ and $g C$. We use the Bruhat decomposition to write $g$ as $g=i_{1} n i_{2}, n \in \mathcal{N}$ and $i_{1}, i_{2} \in \mathcal{G}_{C}$. Denote the product $i_{2} i_{1} \in \mathcal{G}_{C}$ as $k$. Then $\tau\left(1_{g, x}\right)=\tau\left(\operatorname{Ad}\left(i_{1}\right) 1_{n i_{2} i_{1}, x}\right)=\tau\left(1_{n i_{2} i_{1}, x}\right)$, so we may replace $g$ with the element $n k$. In particular, $d_{C}(g)=d_{C}(n)$. Let $z \in C$ satisfy $d_{g}(z)=$ $d_{C}(g)$, and let $F$ be the facet of $C$ containing $z$.

3.5.1. Lemma. Suppose $D$ is a (closed) chamber $D \subset \operatorname{Star}(F) \cap \mathcal{C}(C, g C)$ so that $D \cap[z, g z]$ has positive length. Such a chamber $D$ obviously exists. Then

i) If $C$ and $D$ belong to opposite sides of any affine root hyperplane $H_{\psi} \subset A$ containing $F$, then $D$ and $g C$ belong to the same side of $H_{\psi}$. 
ii) If $\psi$ is an affine root satisfying $\left.\psi\right|_{C} \leq r$ and $\left.\psi\right|_{D} \geq r$, then for every $u \in U_{\psi}$

$$
\operatorname{Ad}(u) g \mathcal{G}_{x, r}=g \mathcal{G}_{x, r} u^{-1} .
$$

Proof. We first prove i). Since $d_{g}$ restricted to $C$ takes a minimal value at the point $z$ which is strictly greater than $d(g)$, it follows from Corollary 2.5 that the intersection of the geodesic $[z, g z]$ with $C$ is precisely the point $z$. If $H=H_{\psi}$ is an affine root hyperplane for which $C$ and $D$ are on opposite sides of $H$, we must have i) $\psi(z)=0$, ii) $\psi(v) \neq 0$ for some point $v \in D \cap[z, g z]$, and iii) there exists $u \in C$ so that $\psi(u)$ and $\psi(v)$ have opposite signs. But then, since the restriction of an affine root to the segment $[z, g z]$ is linear, we conclude that $\psi(g z)$ has the same sign as $\psi(v)$ and so must be in the same half space as $D$. This proves assertion i).

To prove assertion ii), we calculate

$$
\operatorname{Ad}(u) g \mathcal{G}_{x, r}=u n k \mathcal{G}_{x, r} u^{-1}=n k k^{-1} n^{-1} u n k \mathcal{G}_{x, r} u^{-1} .
$$

The element $n^{-1} u n$ belongs to the affine root group $U_{n^{-1}} \psi$. Since $(\psi-r)_{\mid D} \geq 0$ while $(\psi-r)_{\mid C} \leq 0$, i.e. the chambers $C$ and $D$ are on opposite sides of the affine root hyperplane $H_{\psi-r}$, part i) says $(\psi-r)_{\mid n C} \geq 0$, and so $n^{-1} \psi_{\mid C} \geq r$. Thus $U_{n^{-1} \psi} \subset \mathcal{G}_{x, r}$ and so $k^{-1} n^{-1} u n k \in \mathcal{G}_{x, r}$. We conclude $n k k^{-1} n^{-1} u n k \mathcal{G}_{x, r} u^{-1}=$ $g \mathcal{G}_{x, r} u^{-1}$.

3.5.2. Proposition. Suppose $r \in \mathbb{N}(r \geq R$ as in $\S 3.3), A=A(S)$ is an apartment, $C$ is a (closed) chamber in $A, x \in C^{o}$, and $g=n k \in \mathcal{N G}_{C}$ is an element satisfying $d_{C}(g)>d(g)$. Take $D$ to be a chamber as in Lemma 3.5 .1 (so $\left.d_{D}(g)<d_{C}(g)\right)$ and let $p \in D^{o}$. Then, $\tau\left(1_{g, x}\right) \in \tau\left(C_{c}\left(\mathcal{G} / \mathcal{G}_{p, r}\right)_{d_{D}(g)}\right)$.

Proof. It is elementary that a local minimum of a convex function on $\mathcal{B}$ is also a global minimum. Hence, it is a consequence of the hypothesis $d_{C}(g)>d(g)$ that the restriction of the displacement function to $C$ achieves its minimal value on the boundary of $C$. Define $\Gamma$ to be the set of affine roots $\psi$ of $A$ with the property that $\psi_{\mid C} \leq r$ and $\psi_{\mid D} \geq r$. Recall that $\mathcal{G}_{u, r}$ is a product of the affine root groups $U_{\psi}$ satisfying $\psi(u) \geq r$. Let $v$ be an interior point of $D$. Suppose $\psi(v) \geq r$, i.e. $U_{\psi} \subset \mathcal{G}_{v, r}$. Then either $\psi(x)<r$ and so $\psi \in \Gamma$, or $\psi(x) \geq r$ and thus $U_{\psi} \subset \mathcal{G}_{x, r}$. Therefore, we have

$$
\mathcal{G}_{x, r} \prod_{\psi \in \Gamma} U_{\psi}=\mathcal{G}_{x, r} \mathcal{G}_{v, r}
$$

This set, denote it as $J$, is a subgroup of $\mathcal{G}$ and it fixes a neighborhood of $C \cap D$. Thus, for all $k \in J, d_{g k}=d_{g}$ in a neighborhood of the point $z$ (recall $z$ is a point in $C$ so that $d_{g}(z)=d_{C}(g)$ ). Proposition 2.4 implies that there are points $v$ on the geodesic $[z, g z]$ arbitrarily close to $z$ so that $d_{g}(v)<d_{C}(g)$ and $v \in D$. In turn, since $D^{o}$ is dense in $D$, we can find a point $y \in D^{o}$ so that $d_{g}(y)$ is so close to $d_{g}(v)$ that $d_{g}(x)<d_{C}(g)$. Denote as $\psi^{+}$the affine root with the same gradient as $\psi$ and so that the constant $\psi^{+}-\psi$ is the smallest possible positive number. Order the roots of $\Gamma$ in some fashion, choose representatives of $U_{\psi} / U_{\psi^{+}}$and let $u$ run over the ordered product of these representatives. Then, we deduce from Lemma 3.5.1 that

$$
\sum_{u} A d(u) 1_{g, x}:=\text { the characteristic function of } g \mathcal{G}_{x, r} \mathcal{G}_{y, r}=\sum_{h} 1_{g h, y} .
$$


Here, $h$ are representatives for the cosets $\mathcal{G}_{y, r} /\left(\mathcal{G}_{y, r} \cap \mathcal{G}_{x, r}\right)$ and $d_{D}(g h)=d_{D}(g) \leq$ $d_{g}(y)<d_{C}(g)$. Since $\tau\left(A d(u) 1_{g, x}\right)=\tau\left(1_{g, x}\right)$, we conclude that $\tau\left(1_{g, x}\right)$ lies in $\tau\left(C_{c}\left(\mathcal{G} / \mathcal{G}_{y, r}\right)_{d_{D}(g)}\right)=\tau\left(C_{c}\left(\mathcal{G} / \mathcal{G}_{p, r}\right)_{d_{D}(g)}\right)$.

Let $\mathcal{W}$ be a finite set of elements in $\mathcal{G}$ so that if $E$ is any chamber meeting $C$, there exists $w \in \mathcal{W}$ so that $w C=E$.

3.5.4. Corollary. In the setting of Proposition 3.5.2, choose $w \in \mathcal{W}$ so $w C=D$. Then, $d_{C}\left(w^{-1} g h w\right)<d_{C}(g)$ and

$$
\sum_{u} A d(u) 1_{g, x}=\sum_{h} A d(w) 1_{w^{-1} g h w, x} .
$$

Proof. If $X$ is a subset of $\mathcal{G}$, let $1_{X}$ denote the characteristic function of $X$. We have, for $y \in D^{o}$,

$$
\begin{aligned}
1_{g h, y} & =1_{g h \mathcal{G}_{y, r}}=A d(w) 1_{w^{-1} g h \mathcal{G}_{y, r} w}=A d(w) 1_{w^{-1} g h w w^{-1} \mathcal{G}_{y, r} w} \\
& =A d(w) 1_{w^{-1} g h w \mathcal{G}_{w^{-1} y, r}}=\operatorname{Ad}(w) 1_{w^{-1} g h w, w^{-1} y}=A d(w) 1_{w^{-1} g h w, x},
\end{aligned}
$$

and $d_{C}\left(w^{-1} g h w\right)=d_{w C}(g h)=d_{D}(g)<d_{C}(g)$.

3.6. Proof of the Howe Conjecture. We recall that our plan to prove the Howe Conjecture is to show that

$$
\tau\left(C_{c}\left(\mathcal{G} / \mathcal{G}_{x, r}\right)_{s_{m}}\right)=\tau\left(C_{c}\left(\mathcal{G} / \mathcal{G}_{x, r}\right)_{s_{m+1}}\right) \text { when } s_{m} \text { is sufficiently large. }
$$

We have fixed a chamber $C, x \in C^{o}$, and we are considering the functionals $\tau\left(1_{g, x}\right)$ according to two cases: i) $d_{C}(g)=d(g)$, and ii) $d_{C}(g)>d(g)$.

In $\S 3.4$, we showed there are only finitely many nonzero $\tau\left(1_{g, x}\right)$ 's satisfying condition i). Suppose $s_{t} \geq M(\Omega)$. We show that (3.6.1) holds for any $m \geq t$. Suppose that $d_{C}(g)>s_{m+1}$. In case i), (3.6.1) holds by $\S 3.4$. When $g$ satisfies condition ii), Corollary 3.5.4 shows $\tau\left(1_{g, x}\right) \in \tau\left(C_{c}\left(\mathcal{G} / \mathcal{G}_{x, r}\right)_{d^{\prime}}\right)$ for a $d^{\prime}<d_{C}(g)$. Applying finitely many such steps, we conclude that (3.6.1) holds. This completes our proof of the Howe Conjecture.

\section{Weighted orbital INTEGRAlS}

4.1. Our main method also applies to the weighted orbital integrals $J_{\mathcal{M}}$ introduced by Arthur when $k$ is of characteristic zero. We give different proofs of the conjectures in [A1] and [A2].

As some of our arguments assume properties of weighted orbital integrals which Arthur only states for characteristic zero, in this section we assume $k$ has characteristic zero.

Let $K$ be a maximal compact group corresponding to a special point $z \in \mathcal{B}$. Let $\mathcal{P}$ be a parabolic subgroup with Levi decomposition $\mathcal{P}=\mathcal{M N}$. We can and do choose $\mathcal{M}$ so that it contains a maximal split $k$-torus $S$ with $z \in A(S)$. For $f \in C_{c}^{\infty}(\mathcal{G})$ and $\gamma \in \mathcal{M} \cap \mathcal{G}_{\text {reg }}$, the weighted orbital integral $J_{\mathcal{M}}$ is defined to be

$$
J_{\mathcal{M}}^{\mathcal{G}}(\gamma, f):=\int_{\mathcal{G}_{\gamma} \backslash \mathcal{G}} f\left(h^{-1} \gamma h\right) v_{\mathcal{M}}(h) d h .
$$

The notation is as in A1. Following Arthur, we sometimes abbreviate $J_{\mathcal{M}}^{\mathcal{G}}(\gamma, f)$ to $J_{\mathcal{M}}(\gamma, f)$. While $J_{\mathcal{M}}$ is not invariant, it satisfies the following transformation 
property with respect to conjugation:

$$
J_{\mathcal{M}}\left(\gamma, f^{h}\right)=\sum_{Q \in \mathcal{F}(\mathcal{M})} J_{\mathcal{M}}^{\mathcal{M}_{Q}}\left(\gamma, f_{Q, h}\right)
$$

Here $\mathcal{F}(\mathcal{M})$ is the (finite) set of parabolic subgroups containing $\mathcal{M}$, and $f_{Q, h}$ is given by

$$
f_{Q, h}(m):=\delta_{Q}(m)^{\frac{1}{2}} \int_{K} \int_{\mathcal{N}_{Q}} f\left(k^{-1} m n k\right) u_{Q}(k h) d n d k .
$$

4.2. Lemma. Suppose $\mathcal{M}_{Q} \mathcal{N}_{Q}$ is a parabolic subgroup, $S$ is a maximal split $k$ torus in $\mathcal{M}_{Q}$ and $z \in A(S)$ is a special point. Given any $x, y \in A(S)$ and $r \in \mathbb{N}$, there exists $s \in \mathbb{N}$ such that $f \mapsto f_{Q, h}$ maps $C_{c}\left(\mathcal{G} / \mathcal{G}_{x, r}\right)$ into $C_{c}\left(\mathcal{M}_{Q} /\left(\mathcal{M}_{Q}\right)_{y, s}\right)$.

Proof. Choose $s$ and $t$ so that $\left(\mathcal{M}_{Q}\right)_{y, s} \subset \mathcal{G}_{z, t} \subset \mathcal{G}_{x, r}$. Since $K=\operatorname{Stab}(z)$ normalizes $\mathcal{G}_{z, t}$ we have trivially $A d(K)\left(\mathcal{M}_{Q}\right)_{y, s} \subset \mathcal{G}_{z, t} \subset \mathcal{G}_{x, r}$. In particular, for $j \in\left(\mathcal{M}_{Q}\right)_{y, s}$, we have

$$
f_{Q, h}(m j)=\delta_{Q}(m j)^{\frac{1}{2}} \int_{K} \int_{\mathcal{N}_{Q}} f\left(k^{-1} m j n k\right) u_{Q}(k h) d n d k .
$$

Since $\delta_{Q}(m j)=\delta_{Q}(m)$ and

$$
f\left(k^{-1} m j n k\right)=f\left(k^{-1} m j n j^{-1} k k^{-1} j k\right)=f\left(k^{-1} m j n j^{-1} k\right),
$$

the conclusion of the Lemma now follows by a change of variables.

In what follows, $r$ is a fixed large integer, so that $\mathcal{G}_{x, r} \subset K$, and $\Omega$ is a compact subset of $\mathcal{M}$. Denote by $J_{\mathcal{M}}(\Omega)$ the linear space of distributions $f \mapsto J_{\mathcal{M}}(\gamma, f)$ with $\gamma \in \Omega \cap \mathcal{G}_{\text {reg. }}$. In analogy with the invariant orbital integral setting, if $\phi \in C_{c}^{\infty}(\mathcal{G})$, let $\tau(\phi)$ denote the linear functional of $J_{\mathcal{M}}(\Omega)$ whose value on the weighted orbital integral $J_{\mathcal{M}}(\gamma,-)$ is $J_{\mathcal{M}}(\gamma, \phi)$. The analog of Howe's conjecture is the following:

4.3. Theorem ([A2, Corollary 8.4]). Suppose $\Omega$ is a compact subset of a Levi subgroup $\mathcal{M} \subset \mathcal{G}$. The space of linear functionals on the distributions $J_{\mathcal{M}}(\Omega)$ induced by the elements of $C_{c}\left(\mathcal{G} / \mathcal{G}_{z, r}\right)$ is finite dimensional.

Proof. We modify the proof of Theorem 1.4 to show that the space of linear functionals on the distributions $J_{\mathcal{M}}(\Omega)$ induced by $C_{c}\left(\mathcal{G} / \mathcal{G}_{x, r}\right)$ is finite dimensional. Choose a closed chamber $C$ which contains $z$ and let $x \in C^{o}$. Since $C_{c}\left(\mathcal{G} / \mathcal{G}_{z, r}\right) \subset C_{c}\left(\mathcal{G} / \mathcal{G}_{x, r}\right)$, we can and do replace the point $z$ by the point $x$. Again, a necessary condition for the nonvanishing of the distribution $\tau\left(1_{g, x}\right)=\tau_{\mathcal{M}}^{\mathcal{G}}\left(1_{g, x}\right)$ of $J_{\mathcal{M}}(\Omega)$ induced by $1_{g, x}$ is $\mathcal{G} \Omega \cap g \mathcal{G}_{z, r} \neq \varnothing$. As in $\S 3.3$, we filter $C_{c}\left(\mathcal{G} / \mathcal{G}_{x, r}\right)$ by finite-dimensional spaces $C_{c}\left(\mathcal{G} / \mathcal{G}_{x, r}\right)_{s_{i}}$. We consider the same two cases:

i) $d_{C}(g)=d(g)$,

ii) $d_{C}(g)>d(g)$.

Case $d_{C}(g)=d(g)$. This is treated exactly as in $\S 3.4$.

Case $d_{C}(g)>d(g)$. We recall (3.5.5). There is a chamber $D$ adjacent to $C$, which we write as $D=n C$ ( $n$ in the finite set $\mathcal{W})$, with $d_{D}(g)<d_{C}(g)$ so that

$$
\sum_{u} A d(u) 1_{g, x}=\sum_{h} A d(n) 1_{n^{-1} g h n, x} .
$$


The indices $u$ and $h$ run over the sets described in the discussion surrounding (3.5.5). In particular, $d_{C}\left(n^{-1} g h n\right)=d_{D}(g h)=d_{D}(g)<d_{C}(g)$. We apply $J_{\mathcal{M}}(\gamma$, to (4.3.1) and then rewrite both sides using (4.1.2):

$$
\begin{aligned}
\text { LHS } & =\sum_{u} J_{\mathcal{M}}\left(\gamma, 1_{g, x}\right)+\sum_{u} \sum_{Q \neq G} J_{\mathcal{M}}^{M_{Q}}\left(\gamma,\left(1_{g, x}\right)_{Q, u}\right), \\
\text { RHS } & =\sum_{h} J_{\mathcal{M}}\left(\gamma, 1_{n^{-1} g h n, x}\right)+\sum_{h} \sum_{Q \neq G} J_{\mathcal{M}}^{M_{Q}}\left(\gamma,\left(1_{n^{-1} g h n, x}\right)_{Q, n}\right) .
\end{aligned}
$$

We have assumed $r$ is sufficiently large to cause $u \in K$. Then, since $u_{Q}$ is right $K$-invariant we have $\left(1_{g, x}\right)_{Q, u}=\left(1_{g, x}\right)_{Q, 1}$ is independent of $u$. Also, by Lemma 4.2, there is an $s \in \mathbb{N}$ so that for every $Q \in \mathcal{F}(\mathcal{M})$ we have $\left(1_{g, x}\right)_{Q, u} \in$ $C_{c}\left(\mathcal{M}_{Q} /\left(\mathcal{M}_{Q}\right)_{x, s}\right)$. It follows that

$$
\tau_{\mathcal{M}}^{\mathcal{G}}\left(1_{g, x}\right) \in C_{c}\left(\mathcal{G} / \mathcal{G}_{x, r}\right)_{d\left(n^{-1} g n\right)}+\sum_{Q \neq G} \tau_{\mathcal{M}}^{\mathcal{M}_{Q}} \circ()_{Q, 1}+\sum_{w \in \mathcal{W}} \sum_{Q \neq G} \tau_{\mathcal{M}}^{\mathcal{M}_{Q}} \circ()_{Q, w}
$$

The sum over the finite set $\mathcal{W}$ in the right hand side means the subspace

$$
\sum_{Q \neq G} \tau_{\mathcal{M}}^{\mathcal{M}_{Q}} \circ()_{Q, 1}+\sum_{w \in \mathcal{W}} \sum_{Q \neq G} \tau_{\mathcal{M}}^{\mathcal{M}_{Q}} \circ()_{Q, w}
$$

is independent of the element $g$. By induction we may assume for $Q \neq G$ that the space $\tau_{\mathcal{M}}^{\mathcal{M}_{Q}}\left(C_{c}\left(\mathcal{M}_{Q} /\left(\mathcal{M}_{Q}\right)_{x, s}\right)\right)$ is finite dimensional and so (4.3.3) is finite dimensional. We conclude $\tau_{\mathcal{M}}^{\mathcal{G}}\left(C_{c}\left(\mathcal{G} / \mathcal{G}_{x, r}\right)\right)$ is finite dimensional.

4.3.4. Let $\mathcal{C}(\mathcal{G})$ denote the space of Schwartz functions on the group $\mathcal{G}$. Under the usual seminorm topologies, the space $C_{c}^{\infty}(\mathcal{G})$ is dense in $\mathcal{C}(\mathcal{G})$, and Arthur has shown the functionals $J_{\mathcal{M}}(\gamma,-)$ are tempered, i.e. they extend continuously from $C_{c}^{\infty}(\mathcal{G})$ to $\mathcal{C}(\mathcal{G})$. Let $\mathcal{C}\left(\mathcal{G} / \mathcal{G}_{x, r}\right)$ denote the Schwartz functions which are right $\mathcal{G}_{x, r}$ invariant.

4.3.5. Corollary. Suppose $\Omega$ is a compact subset of a Levi subgroup $\mathcal{M} \subset \mathcal{G}$. The space of linear functionals of $\mathcal{C}\left(\mathcal{G} / \mathcal{G}_{z, r}\right)$ spanned by the distributions $J_{\mathcal{M}}(\gamma,-)$ $(\gamma \in \Omega)$ is finite dimensional.

Proof. Let $d:=\operatorname{dim}_{\mathbb{C}}\left(J(\Omega)_{\mid C_{c}}\right)$ denote the dimension of the linear functionals of $C_{c}\left(\mathcal{G} / \mathcal{G}_{z, r}\right)$ induced by the distributions $J_{\mathcal{M}}(\gamma,-)(\gamma \in \Omega)$. Abbreviate $C_{c}\left(\mathcal{G} / \mathcal{G}_{z, r}\right)$, $\mathcal{C}\left(\mathcal{G} / \mathcal{G}_{z, r}\right)$ and $J_{\mathcal{M}}(\gamma,-)$ to $C_{c}, \mathcal{C}$ and $J(\gamma)$ respectively. Since $C_{c} \subset \mathcal{C}$, it follows that $\operatorname{dim}_{\mathbb{C}}\left(J(\Omega)_{\mid \mathcal{C}}\right) \geq d$. We argue by contradiction that $\operatorname{dim}_{\mathbb{C}}\left(J(\Omega)_{\mid \mathcal{C}}\right)=d$. Suppose the dimension is greater than $d$. Choose elements $\gamma_{1}, \ldots, \gamma_{d+1}$ in $\Omega$ so the linear functionals $J\left(\gamma_{1}\right), \ldots, J\left(\gamma_{d+1}\right)$ are independent on $\mathcal{C}$. Choose functions $f_{j} \in \mathcal{C}$ so that $J\left(\gamma_{i}, f_{j}\right)=\delta_{i, j}$ and for each $f_{j}$, take $h_{j, k}$ to be a sequence of functions in $C_{c}$ with the property that $h_{j, k} \rightarrow f_{j}$ as $k \rightarrow \infty$. Then, $J\left(\gamma_{i}, h_{j, k}\right) \rightarrow \delta_{i, j}$ as $k \rightarrow \infty$. As functionals of $C_{c}$, the $J\left(\gamma_{i}\right)$ 's lie in a vector space of dimension $r$, so there exists a nontrivial linear relation: $0=\sum c_{i} J\left(\gamma_{i}\right)$. For $1 \leq j \leq d+1$, this means $0=\sum c_{i} J\left(\gamma_{i}, h_{j, k}\right)$. If we take the limit as $k \rightarrow \infty$, we find $0=c_{j}$. This is a contradiction.

4.4. Arthur uses the weighted orbital integrals to define a larger family of invariant distributions which he denotes $I_{\mathcal{M}}(\gamma)=I_{\mathcal{M}}^{\mathcal{G}}(\gamma)$ (see [A1]). These distributions are 
defined via the weighted character and the recursion formula:

$$
I_{\mathcal{M}}(\gamma, f)=J_{\mathcal{M}}^{\mathcal{G}}(\gamma, f)-\sum_{\mathcal{L} \neq \mathcal{G}} \hat{I}_{\mathcal{M}}^{\mathcal{L}}\left(\gamma, \phi_{\mathcal{L}}(f)\right) .
$$

The sum is over the proper Levi subgroups containing $\mathcal{M}$, and the (weighted character) transform $\phi_{\mathcal{L}}$ takes $f \in \mathcal{C}(\mathcal{G})$ to the function on $\Pi_{\text {temp }}(\mathcal{M})$ given by

$$
\phi_{\mathcal{M}}(f)=\phi_{\mathcal{M}}^{\mathcal{G}}(f): \pi \mapsto J_{\mathcal{M}}(\pi, f) .
$$

In particular, when $\mathcal{M}=\mathcal{G}$, the $\mathcal{G}$-invariant distribution $I_{\mathcal{M}}(\gamma,-)$ is the orbital integral of $\gamma$.

Let $\mathcal{C}\left(\mathcal{G} / / \mathcal{G}_{z, s}\right)$ denote the Schwartz functions of $\mathcal{G}$ which are $\mathcal{G}_{z, s}$ bi-invariant. Arthur conjectured and proved the next result in [A1], [A2]. We finish by deriving it as a corollary to Theorem 4.3.

4.5. Corollary ( $\mathrm{A2}$, Theorem 8.1]). Suppose $\Omega$ is a compact subset of a Levi subgroup $\mathcal{M} \subset \mathcal{G}$. The restriction of the distributions $I_{\mathcal{M}}(\gamma,-), \gamma \in \mathcal{M} \cap \mathcal{G}_{\text {reg }}$, to $\mathcal{C}\left(\mathcal{G} / / \mathcal{G}_{z, r}\right)$ is finite dimensional.

4.5.1. A technical remark. As a preliminary to the proof of Corollary 4.5, we need to combine several results of Arthur.

\subsubsection{Lemma.}

$$
\phi_{\mathcal{M}}^{\mathcal{G}}\left(C_{c}^{\infty}(\mathcal{G})\right) \subset \phi_{\mathcal{M}}^{\mathcal{M}}(\mathcal{C}(\mathcal{M}))
$$

Proof. Suppose $f \in C_{c}^{\infty}(\mathcal{G})$. The map $\pi \rightarrow J_{\mathcal{M}}^{\mathcal{G}}(\pi, f)$ is an analytic function on the space $\Pi_{\text {temp }}(\mathcal{M})$, and in particular a smooth function in the continuous coordinates of $\Pi_{\text {temp }}(\mathcal{M})$. As remarked by Arthur in [A1, it can happen that when $\pi \rightarrow$ $J_{\mathcal{M}}^{\mathcal{G}}(\pi, f)$ is extended to the ambient complex space containing $\Pi_{\text {temp }}(\mathcal{M})$, poles appear; but there are none on $\Pi_{\text {temp }}(\mathcal{M})$ itself. Now, by Arthur's trace PaleyWiener Theorem [A4], a sufficient criterion that a function $\psi$ on the space $\Pi_{\text {temp }}(\mathcal{M})$ be the trace of the operator Fourier Transform is that $\psi$ be compactly supported and smooth in the continuous coordinates. Hence, there exists $f^{\prime} \in \mathcal{C}(\mathcal{M})$ such that $J_{\mathcal{M}}^{\mathcal{G}}(\pi, f)=\operatorname{tr}\left(\pi\left(f^{\prime}\right)\right)=J_{\mathcal{M}}^{\mathcal{M}}\left(\pi, f^{\prime}\right)$.

4.5.3. Proof of Corollary 4.5. We use induction on the difference in the $k$-ranks of $\mathcal{G}$ and $\mathcal{M}$. If $\mathcal{M}=\mathcal{G}$, then $I_{\mathcal{M}}(\gamma,-)=J_{\mathcal{M}}(\gamma,-)$ and the assertion of finite dimensionality follows from Corollary 4.3.5. Assume Corollary 4.5 is true for all proper Levi subgroups $\mathcal{L} \subsetneq \mathcal{G}$ containing $\mathcal{M}$. For the pair $\mathcal{G}$ and $\mathcal{M}$, it is enough to show finite dimensionality for the space $C_{c}\left(\mathcal{G} / / \mathcal{G}_{z, r}\right)$; the extension to $\mathcal{C}\left(\mathcal{G} / / \mathcal{G}_{z, r}\right)$ follows by a trivial modification of the proof of Corollary 4.3.5. Let $\mathcal{C}\left(\mathcal{M} / / \mathcal{M}_{z, s}\right)$ denote the Schwartz functions of $\mathcal{M}$ which are $\mathcal{M}_{z, s}$ bi-invariant. The key property needed to establish Corollary 4.5 is the following:

$$
\phi_{\mathcal{M}}^{\mathcal{G}}\left(C_{c}\left(\mathcal{G} / / \mathcal{G}_{z, r}\right)\right) \subset \phi_{\mathcal{M}}^{\mathcal{M}}\left(\mathcal{C}\left(\mathcal{M} / / \mathcal{M}_{z, r+1}\right)\right) .
$$

We establish property (4.5.4) by contradiction. Suppose there exists $f \in C_{c}\left(\mathcal{G} / / \mathcal{G}_{z, r}\right)$ with $\phi_{\mathcal{M}}^{\mathcal{G}}(f)$ not in $\phi_{\mathcal{M}}^{\mathcal{M}}\left(\mathcal{C}\left(\mathcal{M} / / \mathcal{M}_{z, r+1}\right)\right)$. Take, by Lemma $4.5 .2, f^{\prime} \in \mathcal{C}(\mathcal{M})$ so that $\phi_{\mathcal{M}}^{\mathcal{G}}(f)=\phi_{\mathcal{M}}^{\mathcal{M}}\left(f^{\prime}\right)$. The hypothesis $\phi_{\mathcal{M}}^{\mathcal{M}}\left(f^{\prime}\right) \notin \phi_{\mathcal{M}}^{\mathcal{M}}\left(\mathcal{C}\left(\mathcal{M} / / \mathcal{M}_{z, r+1}\right)\right)$ means there exists a $\pi \in \Pi_{\text {temp }}(\mathcal{M})$ which does not have any nonzero $\mathcal{M}_{z, r+1}$-fixed vectors for which $0 \neq \phi_{\mathcal{M}}^{\mathcal{M}}\left(f^{\prime}\right)(\pi)=\phi_{\mathcal{M}}^{\mathcal{G}}(f)(\pi)=J_{\mathcal{M}}(\pi, f)$. Now

$$
J_{\mathcal{M}}(\pi, f)=\operatorname{tr}\left(\mathcal{R}_{\mathcal{M}}(\pi, \mathcal{P}) \mathcal{I}_{\mathcal{P}}(\pi, f)\right)
$$


where $\mathcal{I}_{\mathcal{P}}(\pi)$ is the induced representation $\operatorname{Ind}_{\mathcal{P}}^{\mathcal{G}}(\pi)$. The operator $\mathcal{I}_{\mathcal{P}}(\pi, f)$ ), hence the trace, is zero unless $\mathcal{I}_{\mathcal{P}}(\pi)$ has nonzero $\mathcal{G}_{z, r}$-fixed vectors. But if any constituent of $\operatorname{Ind}_{\mathcal{M}}^{\mathcal{G}}(\pi)$ has a nonzero $\mathcal{G}_{z, r}$-fixed vector, this would imply, using the Jacquet functor and $\mathrm{MP}$. Theorem 4.5], that the depth of $\pi$ is less than $r$ and thus it has a nonzero $\mathcal{M}_{z, r+1}$-fixed vector. This contradicts the assumption about $\pi$.

Having established (4.5.4), the finite dimensionality of the $\mathcal{G}$-invariant distributions $I_{\mathcal{M}}^{\mathcal{G}}(\gamma,-)$ on the space $C_{c}\left(\mathcal{G} / / \mathcal{G}_{x, r}\right)$ follows easily from Theorem 4.3 , the recursion formula (4.4.1) and our induction hypothesis.

\section{ACKNOWLEDGEMENTS}

The authors thank James Arthur, Stephen De Backer, Samuel Evens, Thomas Hales, Roger Howe, Ju-Lee Kim, and Fiona Murnaghan for discussions during the period this paper was written. The authors also thank the referee for some useful comments.

\section{REFERENCES}

[A1] J. Arthur, Some problems in local harmonic analysis, in Harmonic Analysis on Reductive Groups, W. Barker and P. Sally, eds., Birkhäuser, 1991. MR 93e:22034

[A2] - On the Fourier transforms of weighted orbital integrals, J. Reine Angew. Math. 452 (1994), 163-217. MR 95h:22015

[A3] - Intertwining operators and residues I. Weighted characters, J. Funct. Anal. 84 (1989), 19-84. MR 90j:22018

[A4] Trace Paley-Wiener Theorem for Schwartz functions, in Representation Theory and Analysis on Homogeneous Spaces, AMS Contemp. Math. 177 (1994), 171-180. MR 95k:22010

[BT] F. Bruhat and J. Tits, Groupes réductifs sur un corps local I, Publ. Math. I. H. E. S. 41 (1972). MR 48:6265

[C] L. Clozel, Orbital integrals on p-adic groups: a proof of the Howe conjecture, Ann. of Math. 129 (1989), 237-251. MR 90h:22020

[HC] Harish-Chandra, Admissible invariant distributions on reductive p-adic groups, Preface and notes by S. DeBacker and P. Sally, University Lecture Series, vol. 16, American Mathematical Society, Providence, RI, 1999. CMP 99:16

[Ho1] R. Howe, Two conjectures about reductive p-adic groups, Proc. of A.M.S. Symposia in Pure Math. XXVI (1973), 377-380. MR 49:3044

[Ho2] _ The Fourier transform and germs of characters, Math. Annalen 208 (1974), 305322. MR 49:7391

$[\mathrm{M}] \quad$ A. Moy, Displacement functions on the Bruhat-Tits building, preprint.

[MP] A. Moy and G. Prasad, Jacquet functors and unrefined minimal K-types, Comment. Math. Helvetici 71 (1996), 98-121. MR 97c:22021

Department of Mathematics, Cornell University, Malott Hall, Ithaca, New York 14853-4201

E-mail address: barbasch@math.cornell.edu

Department of Mathematics, University of Michigan, Ann Arbor, Michigan 481091109

E-mail address: moy@math.1sa.umich.edu 